\title{
QoS class-based proportional resource allocation for LTE downlink
}

\begin{abstract}
In LTE multi-service communication system, a trade-off between QoS assurance and fairness is a challenging issue, since the QoS provisioning at the cost of starving users in low service demand classes is not favorable for the operator. In this paper, we adopt the time-domain Knapsack algorithm and fine tune it to provide fair resource allocation while support QoS requirements in LTE downlink scheduling system when the bearers are from different classes of service, having different QoS characteristics. We demonstrate that more efficient performance can be achieved in two aspects of fairness and QoS provisioning in terms of normalized throughput, and packet loss and delay rate, which are evaluated using simulation results.
\end{abstract}

Keyword: Downlink resource allocation; Fairness; Knapsack algorithm; LTE networks; QoS 